\title{
Traffic Flow Prediction Based on the location of Big Data
}

\author{
Xijun Zhang, Zhanting Yuan \\ Lanzhou Univ Technol, Coll Elect \& Informat Engn \\ Lanzhou, China \\ zhangxijun198079@sina.com
}

Keywords: Traffic big data; intelligent transportation; smart city

Abstract: This article focuses on the design of intelligent transportation systems in the construction of the smart urban. Learning from the wisdom of traffic building programs, the paper is given the overall design of intelligent transportation process based on location big data. Lanzhou City was analyzed and designed using this method. The collected GPS big data was deeply excavated and analyzed through the mathematical model. Thus the clear city traffic analysis chart was got to predict the traffic flow. In the end the intelligent transportation was realized in the smart city.

\section{INTRODUCTION}

The arrival of the "smart city" is not only changing the quality of life of personal information, but also can be applied to urban public safety, manufacturing production, environmental monitoring, intelligent traffic, intelligent home, environmental monitoring and other fields. It can make full use of the social resources. It can greatly promote the enterprise to reduce the cost. It can make the government improve public service ability and city management efficiency.[1-3] From the point of the architecture of the smart city the wisdom of the city is based on the technology of the Internet of things, so the architecture of the smart city is similar to the internet of things. It is divided into four layers which are the perception layer, transport layer, system layer and application layer.

The goal of the intelligent transportation system (ITS) is using the communication system to closely combine the transport system of people, vehicles and road. Advanced traffic control system and advanced traffic management system are required to provide real-time traffic flow information. Traffic flow analysis is an important research content in the transportation system. The traditional traffic flow model is described the traffic flow state variables (velocity, density and flow) with the change of time and space.[4-5] From the research of traffic flow, the research method of traffic flow is developed from the empirical method to the research method based on physical model and the mathematical model. Finally it returns to the initial empirical method.The Fig.1 is shown the platform of the location big data.[6-8] All study is based on the data. The location big data is studied through this platform. There are four steps which are data acquisition, data analysis, data calculation and data visualization. The intelligent transportation on business analysis can be got through those process. 


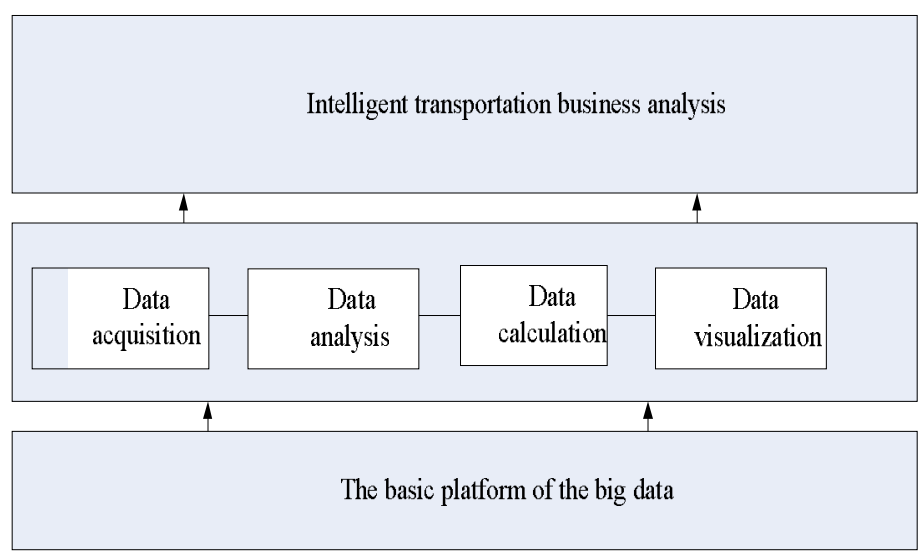

Fig.1. The platform of the location big data

\section{The way of studying methods}

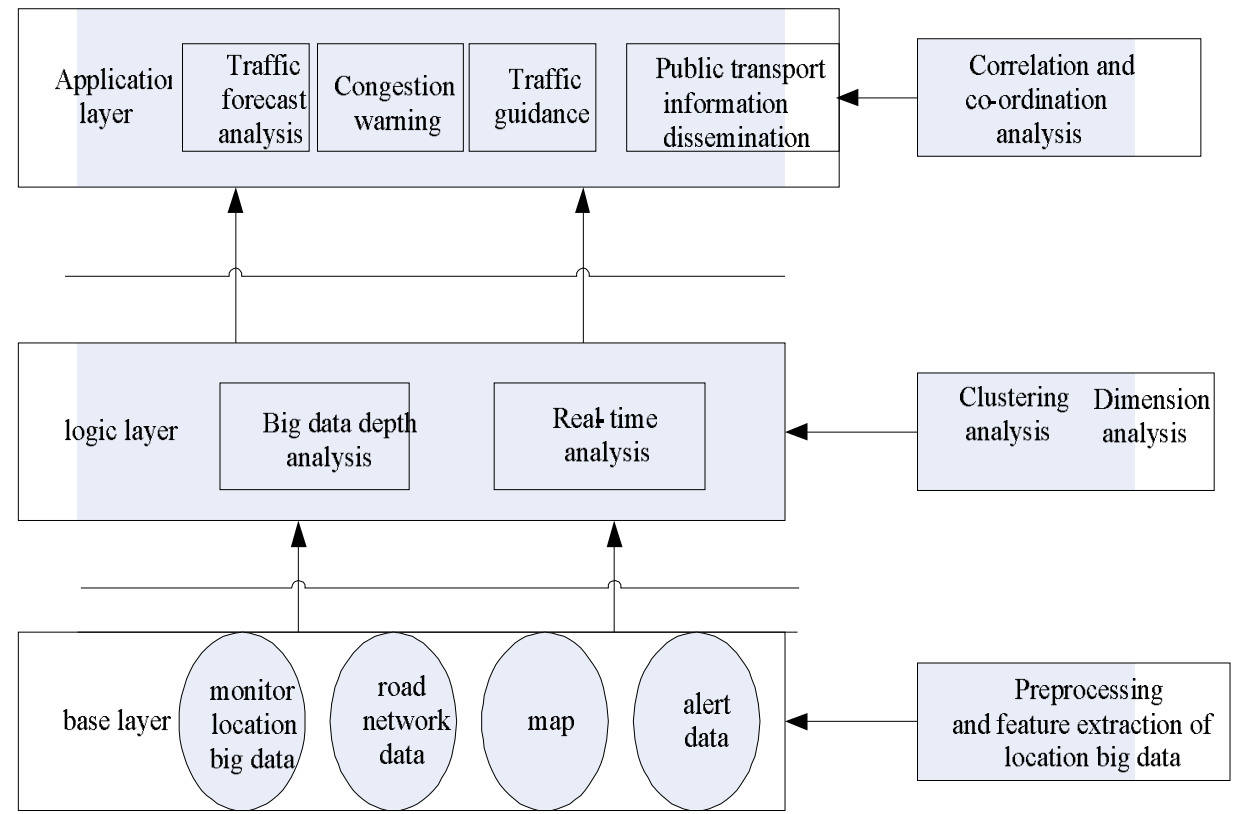

Fig.2. The study content

Intelligent transportation system (ITS) is a very large system. Because the ITS has the character of complexity and stability, the quality of traffic data acquisition is difficult to guarantee. The architecture diagram for the study is shown in Fig.2. Location big data especially trajectory data analysis, the general requirements of the data set with a higher sampling rate, so it often need a simple interpolation of trajectory data, namely the use of effective interpolation algorithm of missing or unknown trajectory to repair, to recover the complete trajectory curve. According to the interpolation algorithm there are linear interpolation and nonlinear interpolation. At present, there are a lot of interpolation algorithms including the inverse distance weighted interpolation method, nearest neighbor interpolation method, moving average method, local polynomial method and so on. The performance of trajectory reconstruction is compared with the performance of different interpolation algorithms, so the best method of data preprocessing is found to lay the foundation for the forecast of traffic flow.

\section{Trajectory reconstruction of the vehicle}

Taking the time and space location big data as an example, the vehicle's track data missing in the time $\left[t_{1}, t_{1}+\tau\right]$ because of the transmission malfunction and so on. Thus, the single vehicle 
trajectory reconstruction is designed to use the interpolation algorithm to make up the missing sampling points, so the full trajectory of the vehicle can be recovered. If the speed and acceleration of the vehicle in the time $\left[t_{1}, t_{1}+\tau\right]$ are also missing, the local polynomial method can be used to merge interpolation. Suppose the snapshot data of the vehicle is collected periodically. The time interval is $\tau$ and selected data points are $\mathrm{k}$, and then the $2 \mathrm{k}-1$ sub polynomial is constructed.

$$
x(t)=\sum_{i=0}^{2 k-1} a_{i} t^{i}
$$

As is shown in equation (1) $a_{i}(i=0,1, \mathrm{~L}, 2 k-1)$ is the undetermined coefficient. According to the $2 \mathrm{k}$ known points the values of the corresponding positions of the missing time points can be recovered.If the speed and acceleration of the vehicle are known within the time $\left[t_{1}, t_{1}+\tau\right]$. The speed is $\mathrm{v}(\mathrm{t})=\frac{\mathrm{dx}(\mathrm{t})}{\mathrm{dt}}$, and the location is $x_{k}$ :

$$
\mathrm{x}_{\mathrm{k}}=x_{1}+\int_{t_{1}}^{t_{p}} v(t) d t
$$

In the formula (2): the expression for the position to be found is $x_{k}$. Thus, the vehicle can be calculated in the missing trajectory segment.

\section{Reconstruction of whole space-time trajectory}

The time to the position trajectory can be extended to the three-dimensional space to increase the speed or density variable. We can obtain the three-dimensional vehicle trajectory. The reconstruction of the whole space-time trajectory is based on the vehicle trajectory and used the correlation interpolation algorithm to reconstruct the unknown trajectories in the space. The steps of trajectory reconstruction algorithm is as follows: we select $\Delta t$ and $\Delta x$ to time position plane grid, We use nearest neighbor algorithm, anti distance weighted algorithm or moving average interpolation to calculate the grid on each point corresponding to the velocity values and reconstruct the velocity curved surface.

\section{The characteristic parameters extraction used wavelet transform}

The primary analysis of the location big data is extracted the value from the local subjects, so it is namely the initial feature extraction. In traffic flow theory the traffic characteristics may be described and analyzed from the micro and macro two angles. The nature of wavelet de-noising problem is a function approximation problem. To find the best approximation of the original signal to complete distinction between the original signal and the noise signal. Wavelet de-noising is a signal filtering problem. The wavelet de-noising can be seen as a low-pass filter, but after de-noising it can also successfully retain signal characteristics. For this reason the wavelet method is better than the traditional low-pass filter. The principle is shown in Fig.3.

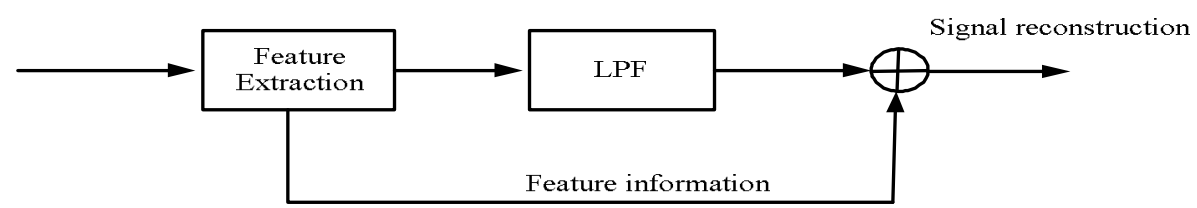

Fig.3. The figure of wavelet de-noising 


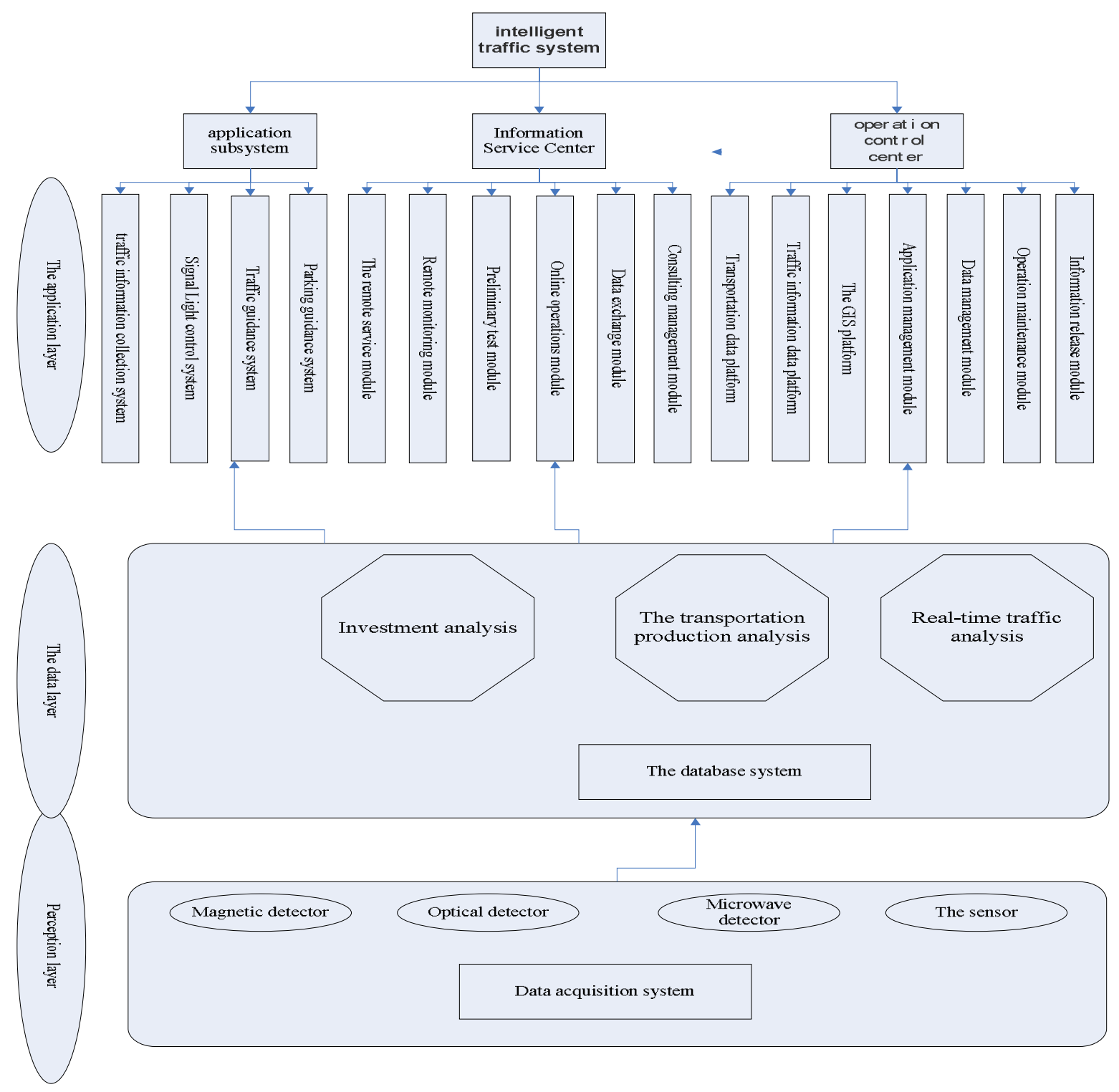

Fig. 4 the designed scheme of intelligent transportation system

The smart city of Lanzhou is mainly studied from the data layer research. We studied the traffic operation status from the aspects of investment and real-time traffic to provide decision for the managers. Designed scheme of intelligent traffic system is shown in Fig.4.

As is shown in the figure, the design of the intelligent traffic system can be divided into three layers to build: perception layer, data layer and application layer. Perception layer includes a data acquisition system, which is mainly using a variety of detectors and sensors for detection of real-time traffic; data layer includes database system, which involves the investment on traffic analysis, transport production analysis and real-time traffic analysis; application layer mainly includes the application of intelligent traffic processing layer. It is responsible for the analysis of traffic big data processing, traffic control and guidance system, so convenient transportation wizard is provided for people. 


\section{Conclusion}

Project supported by the National Natural Science Foundation of China(61461027).

Intelligent transportation is in the field of transportation to take advantage of networking, space perception, cloud computing, mobile Internet and other new generation of information technology. It is taken the big data as the core technology to form problem analysis model. To promote the operation and development of transportation safer, more efficient, more convenient, more economical and more comfortable driving ways the study of location big data is wildly studied.

\section{References}

[1] Making sense of Big Data. Technology forecast, A quarterly journal, 2010.

[2] Liu JN. The recent progress on high precision applications of Beidou navigation satellite system. Report of the Stanford's 2012 PNT Challenges and Opportunities Symp. (SCPNT 2012), 2012.

[3] Guo C, Fang Y, Liu JN, Wan Y. Study on social awareness computation methods for location-based services. Journal of Computer Research and Development, 2013,50(12):2531-2542.

[4] Yuan NJ, Zheng Y, Zhang LH, Xie X. T-Finder: A recommender system for finding passengers and vacant taxis. IEEE Trans. On Knowledge and Data Engineering, 2012,25(10):2390-2403.

[5] Chawla S, Zheng Y, Hu J. Inferring the root cause in road traffic anomalies. In: Proc. of the IEEE 12th Int'l Conf. on Data Mining (ICDM). Piscataway: IEEE, 2012. 141-150.

[6] Pan G, Qi GD, Wu ZH, Zhang DQ, Li SJ. Land-Use classification using taxi GPS traces. IEEE Trans. on Intelligent Transportation Systems, 2012, 14(1):113-123.

[7] Clifford Lynch. Big data: How do your data grow? [J]. Nature, 2008, 455(7209) :28 -29.

[8] Paul.C, Chris Eaton, Dirk Deroos etc. Understanding big data analytics for Enterprise Class Hadoop and streaming data [M]. McGraw Hill, 2012. 